\title{
Correction: The potent and selective cyclin-dependent kinases 4 and 6 inhibitor ribociclib (LEE011) is a versatile combination partner in preclinical cancer models
}

\section{Sunkyu Kim ${ }^{1}$, Ralph Tiedt ${ }^{2}$, Alice Loo ${ }^{1}$, Thomas Horn ${ }^{1}$, Scott Delach ${ }^{1}$, Steven Kovats ${ }^{1}$, Kristy Haas $^{1}$, Barbara Schacher Engstler ${ }^{2}$, Alexander Cao ${ }^{1}$, Maria Pinzon-Ortiz ${ }^{1}$, Iain Mulford ${ }^{1}$, Michael G. Acker ${ }^{1}$, Rajiv Chopra ${ }^{3}$, Christopher Brain ${ }^{4}$, Emmanuelle di Tomaso ${ }^{1}$, William R. Sellers ${ }^{1}$ and Giordano Caponigro ${ }^{1}$}

\footnotetext{
${ }^{1}$ Novartis Institutes for BioMedical Research, Oncology Disease Area, Cambridge, MA, USA

${ }^{2}$ Novartis Institutes for BioMedical Research, Oncology Disease Area, Basel, Switzerland, USA

${ }^{3}$ Novartis Institutes for BioMedical Research, Chemical Biology \& Therapeutics, Cambridge, MA, USA

${ }^{4}$ Novartis Institutes for BioMedical Research, Global Discovery Chemistry, Cambridge, MA, USA
}

Published: April 07, 2020

Copyright: Kim et al. This is an open-access article distributed under the terms of the Creative Commons Attribution License 3.0 (CC BY 3.0), which permits unrestricted use, distribution, and reproduction in any medium, provided the original author and source are credited.

This article has been corrected: In Table 1, a unit of measurement is displayed incorrectly. " $\mu \mathrm{M}$ " is used instead of " $\mathrm{MM}$ ". The corrected Table 1 is shown below. The authors declare that these corrections do not change the results or conclusions of this paper.

Original article: Oncotarget. 2018; 9:35226-35240. https://doi.org/10.18632/oncotarget.26215

Table 1: $\mathrm{IC}_{50}$ Values of CDK4/6 Inhibitors

\begin{tabular}{lccccc}
\hline Cell line & Cancer type & Dominant CDK & $\begin{array}{c}\text { Ribociclib IC } \\
\text { mean } \pm \text { SD, } \mathbf{n M}\end{array}$ & $\begin{array}{c}\text { Palbociclib IC }_{\mathbf{5 0}}, \begin{array}{c}\text { Abemaciclib IC } \\
\text { mean } \pm \text { SD, } \mathbf{n M}\end{array} \\
\text { mean } \pm \text { SD, nM }\end{array}$ \\
\hline JeKo-1 & MCL & CDK4 & $143 \pm 87$ & $72 \pm 33$ & $20 \pm 9$ \\
CAMA-1 & ER+ BC & CDK4 & $162 \pm 59$ & $50 \pm 24$ & $28 \pm 2$ \\
MCF-7 & ER+ BC & CDK4 & $62 \pm 30$ & $30 \pm 18$ & $11 \pm 7$ \\
T47D & ER+ BC & CDK4 & $111 \pm 14$ & $66 \pm 19$ & $13 \pm 3$ \\
REH & ALL & CDK6 & $1030 \pm 246$ & $60 \pm 17$ & $72 \pm 6$ \\
SEM & ALL & CDK6 & $1484 \pm 215$ & $87 \pm 28$ & $162 \pm 37$ \\
Pfeiffer & DLBCL & CDK6 & $948 \pm 53$ & $89 \pm 32$ & $66 \pm 25$ \\
MOLM-13 & AML & CDK6 & $365 \pm 62$ & $47 \pm 25$ & $57 \pm 21$ \\
\hline
\end{tabular}

$\mathrm{IC}_{50}$ values (mean $\pm \mathrm{SD}$ ) of ribociclib, palbociclib, and abemaciclib were determined using the CyQuant cell proliferation assay. The average differential for CDK4 versus CDK6 dependent lines for ribociclib, palbociclib, and abemaciclib is 8.0-, 1.3-, and 5.5-fold, respectively. Abbreviations: ALL, acute lymphoblastic leukemia; AML, acute myeloid leukemia; BC, breast cancer; CDK, cyclin-dependent kinase; DLBCL, diffuse large B-cell lymphoma; ER+, estrogen receptor-positive; IC ${ }_{50}$, halfmaximal inhibitory concentration; MCL, mantle-cell lymphoma; SD, standard deviation. 